\title{
Wavelet Transform in Vibroacoustic Diagnostics of Combustion Engines
}

\author{
Radoslaw Wrobel*, Andrzej Kazmierczak \\ Wroclaw University of Science and Technology, Wroclaw, Poland \\ Email: *radoslaw.wrobel@pwr.edu.pl
}

How to cite this paper: Wrobel, R. and Kazmierczak, A. (2019) Wavelet Transform in Vibroacoustic Diagnostics of Combustion Engines. Engineering, 11, 395-406. https://doi.org/10.4236/eng.2019.117028

Received: May 24, 2019

Accepted: July 14, 2019

Published: July 17, 2019

Copyright $\odot 2019$ by author(s) and Scientific Research Publishing Inc. This work is licensed under the Creative Commons Attribution International License (CC BY 4.0).

http://creativecommons.org/licenses/by/4.0/

\begin{abstract}
This article discusses the use of wavelet decomposition in the diagnostics of vibrometric signals of an engine. Apart from presenting the possibility of using wavelets in diagnostics, the authors take up the subject of the applicability range of processing for stationary signals, which until now has been reserved for non-stationary signals. A unified definition of signal stationarity has been proposed, which is not based on statistics. The authors presented methods of wavelet decomposition of a vibrometric signal of combustion engine vibrations, measured with the use of LDV (Laser Doppler Vibrometry). Laser measurements allow for studying an object without "touching" its housing. Basing on the relative velocity of engine vibrations, the authors indicate how reliable vibrations are in diagnostics. Despite higher costs, this measurement method gives better results (for specific cases) than acoustic studies. Transform-wavelet decomposition is a solution hardly ever used in machine diagnostics; it is more often applied in medicine and image recognition. The authors presented the differences that can be obtained for different levels of decomposition, and also presented the impact on the engine condition assessment through the use of filtering (windowing) the signal before decomposition.
\end{abstract}

\section{Keywords}

DSP, Wavelet, LDV, Combustion Engine

\section{Introduction}

In 1992, a book by Ingrid Daubechies was published [1], which was to become the cornerstone of practical application of wavelet transformation for discrete signals. In her work, the author presents a series of wavelets (mainly orthogonal and biorthogonal), the use of which has become easy along with increasing computing power. What stands out here are especially widely used non-symmetrical 
wavelets as well as finite carrier wavelets [2].

The wavelet transform, like the more popular (discrete) Fourier transform, relies on a multiplication operation between the input signal and the transformation function, often referred to as the kernel [2] [3]. Because the Fourier transform makes use of sinusoidal functions (with undefined duration), information about the phase of the signal after transformation disappears [2] [4]. This is the feature that points to the use of the transformation in machine diagnostics. For instance, information about the top dead centre (TDC) of the piston in the engine is not required if a vibroacoustic diagnostic signal is the research subject. In the case of wavelets, the transformation kernel is a function (wavelet) with finite duration. Therefore, information about the signal phase is preserved, and its resolution depends on the size of the wavelet [2] [3] [4].

It follows from the above considerations that the Fourier transform is ideally suited for the analysis of stationary diagnostic signals, while the wavelet transform is most suitable for non-stationary signals. The definition of stationarity and non-stationarity signals is particularly problematic. The division (into stationary and non-stationary) results from (statistical) research by N. Weiner and N.A. Kolmogorov, who stated in the 1940s that a stationary signal is one whose "statistics" do not change during observation [5]. Their approach was reasonable as far as implementation possibilities of particular transforms are considered. Since then, the computing power has increased immensely, and thus the Fourier transform, Wigner-Ville or wavelet transform is no longer a problem in terms of diagnostic systems implementation. The question is (for instance) whether a diagnostic vibroacoustic signal of combustion engine vibrations (regardless of the measurement method) is stationary by nature? Does a damaged engine, whose vibration spectrum has changed, produce a non-stationary signal due to the occurrence of the defect? Conventionally, statistical approach to the task would be required: a number of exactly the same engines would be tested under the same conditions with use of the same equipment. In a book published in 1996 [6], the authors explicitly state that some types of signals, despite the lack of randomness in duration, should be treated as non-stationary (they give here the example of sounds produced by birds).

It should be noted that stationary influences are also very important opposite the dynamic signals, such as temperature influence and compensation, and other influences such as parasitic capacitances, inductances or resistances which have been described in detail in the literature [7] [8].

Currently, Doppler Laser Vibrometry can always show some differences in speed or relative displacement of vibrations in every copy of the same type of engine (even if it comes from one factory). Discrete Fourier Transform is often used in diagnostics of engines (systems and machines) to locate damage or predict its occurrence [5]. However, by definition, an engine damaged in this way will vibrate differently from a group of correctly operating machines of the same model. Accordingly, it is possible to use the analysis by definition intended for stationary signals in (statistically) non-stationary signals. The authors suggest, 
therefore, to employ a new definition of the non-stationary nature of signals: 'the non-stationary signal is one which is different from the signal coming from an object of the same type (model), which cannot become the basis for diagnostic comparative analysis. It follows from the definition that the vast majority of diagnostic signals are stationary, and that the use of transformations, conventionally designed for non-stationary signals, allows for obtaining valuable diagnostic information. The wavelet transform for vibroacoustic spectrum of an engine can be this kind of transformation.

In one of the reviews (critical reviews as the authors claim) [9] regarding applications of vibroacoustics, it was shown how few elements are currently diagnosed with the use of vibroacoustics. It was found that gears and bearings are "the easiest" to diagnose, while diagnostics of other elements is much more difficult because it requires more operations. It appears crucial to indicate the measurement method, which seems to have often been overlooked in the literature thus far. Despite the fact that displacement, velocity and acceleration are derivatives, they provide additional "information" (such as the constant component), which should be filtered out.

\section{Methods of Measurement and Processing}

The properties of vibroacoustic signals, often wrongly considered as synonymous with vibrations, are widely described in literature [10]-[18]. However, until now, the definition of signals or vibroacoustic vibrations has not been unified. It should be assumed that vibroacoustic vibrations carry a narrower meaning concerning one specific medium. A complex of issues is meant by vibroacoustic signal, i.e. both vibrations and the measured discrete representation of these vibrations, and in particular their characteristic values.

Mechanical vibration is a phenomenon involving the transformation of kinetic energy into potential energy, which is further transformed into kinetic energy, etc., until the phenomenon dies down [11] [14]. The measurement of mechanical vibrations depends on the number of degrees of the system freedom.

Attention should be paid to the large complexity of vibration diagnostics, since a mechatronic system, such as an internal combustion engine, has a large number of degrees of freedom. To avoid a large number of calculations, physical systems are interpolated unto those in which the number of degrees of freedom is known. In such systems, the elements with the lowest mass are accepted as deformable bonds, while elements with a larger mass are treated as material points or rigid bodies [11] [14].

An acoustic signal is a disturbance, consisting in the creation of a sound source under the influence of vibrations with sufficient energy to induce a human auditory sensation [19]. This disorder was first described by Isaac Newton [10] as a rapid change in pressure and air vibrations. This means that the same density of air in each point of space is identical to silence. Changes in air density, resulting in changes in sound pressure, i.e. disturbances in atmospheric pressure, are called acoustic density [19]. In addition to atmospheric pressure disturbances, 
the acoustic wave is accompanied by a temperature disturbance of the medium. A full description of the acoustic wave should include all parameters characterizing it (acoustic density, acoustic pressure, acoustic temperature, acoustic particle deflection, velocity of the acoustic particle, acceleration of the acoustic particle).

Authors [11] [13] define vibroacoustic signals as a whole of dynamic phenomena-mechanoacoustic, which take place in machines, devices and special constructions. These phenomena are vibrations, noise, air and material sound as well as medium pulsations in machine working spaces. They occur in a wide frequency range from very low, almost zero, to very high frequencies $(\mathrm{MHz})$.

Considering the above-mentioned definitions, it can be concluded that vibroacoustic vibrations are phenomena consisting in the motion of the material system around a static equilibrium point, which in turn consists in the flow and storage of energy with a sufficient value to create a sound source of sufficient intensity to induce a human auditory sensation, but at the same time capable of exceeding the range of frequencies heard by people.

Vibroacoustic vibrations are often mistakenly used synonymously with the values marking them. The following physical quantities are used to quantify the vibration process [19] [20]:

- displacement,

- relative displacement,

- velocity,

- relative velocity,

- acceleration,

- harmonic phase shift.

The quantities that characterize vibroacoustic vibrations are measured by various methods, which include piezoelectric acceleration and laser vibrometry (LDV), which is recently very popular. The measurement method is closely related to the measured quantity. In the case of accelerometry, relative acceleration is measured directly, while displacement and relative velocity are measured in the case of vibrometry. Other quantities are measured in diagnostic systems; however, they result from transformations of basic quantities.

While performing vibroacoustic vibration measurements, it is assumed that the system is continuous, and this means a determined number of points of freedom and, moreover, the necessity to move from discretization, based on differential equations, to continuity, based on integer calculus [20]. It can be concluded that vibroacoustic vibrations measurements are an approximation at the detection level, and the discretisation of the system is approximated by continuity.

Wavelet transformation is based on a linear combination of scalable and shifting functions. It can therefore be interpreted as the filtering of the signal analysed by the analysing function. The analysing function must be marked by an appropriate location in the time domain and by a limited band [1] [21] [22]

It is impossible to use the continuous transform in engineering applications; 
this is why, a discrete representation of wavelets is employed. The discreet wavelet representation, re-scaled at the m-level, with the $\mathrm{n}$-shift is recorded as [2]:

$$
\Psi_{a, b}(t) \rightarrow \Psi_{m, n}(t)=(2)^{-m / 2} \Psi\left(2^{-m} t-n T\right)
$$

Starting from the analogue version of the transform [2] [17]:

$$
W_{x}(a, b)=|a|^{-1 / 2} \int_{-\infty}^{\infty} x(t) \Psi\left(\frac{t-b}{a}\right) \mathrm{d} t
$$

where: $\Psi$-wavelet function, $a$-time scale, $b$-displacement.

The discrete wavelet transform can be written as a convolution function of the analysed signal $x(t)$ and the wavelet function $\Psi(t)$ [2]:

$$
W_{x}\left(2^{m}, b\right)=2^{-m / 2} \int_{-\infty}^{\infty} x(t) \Psi\left[2^{-m}(t-b)\right] \mathrm{d} t
$$

The discrete wavelet transform is reversible, which, however, is burdened with certain additions resulting from the discretization of the signal.

If the wavelet transform is treated as a function used to emphasize the changes occurring in the signal, then the usefulness of wavelets in diagnostics of non-stationary signals seems obvious. The value of such emphasis or "highlight" is a derivative of superimposed (multiharmonic) vibration signals, which are produced by moving parts of the engine and the combustion process. The degree of "emphasis" indicates the technical condition of the engine elements (moreover, it is possible to interpret those parts of the signal that will not be "emphasized", but it is more difficult).

Currently, Discrete Wavelet Transform (DWT) in engineering applications is treated as a method of recursive filtering of the source diagnostic signal, in accordance with formula (3).

\section{Resaults of Vibroacoustic Tests of the Technical Condition of an Internal Combustion Engine with the Use of Wavelet Transformation}

The research object was an extremely popular self-ignition engine with a capacity of $1900 \mathrm{ccm}$, produced from 1991 to 2012 (its successor, equipped with an improved power system and raised capacity volume up to $2000 \mathrm{ccm}$, is manufactured today). The engine had been constantly developed; initially, the power system was based on a rotary pump, later on unit injectors, and in the final phase on a common rail system. The elements that were most often subject to damage were among others EGR system (Exhaust Gas Recirculation), injectors or turbocharger. The following figure shows the engine vibration using LDV analysis. The measured variable was relative velocity, which excludes the constant component (as is the case of measuring displacements).

The direct measurement of the vibration speed itself is extremely valuable diagnostic information. You can see the course of the combustion process (Figure 1); number 1 indicates pre-injection, number 2 injection proper. Additionally, 


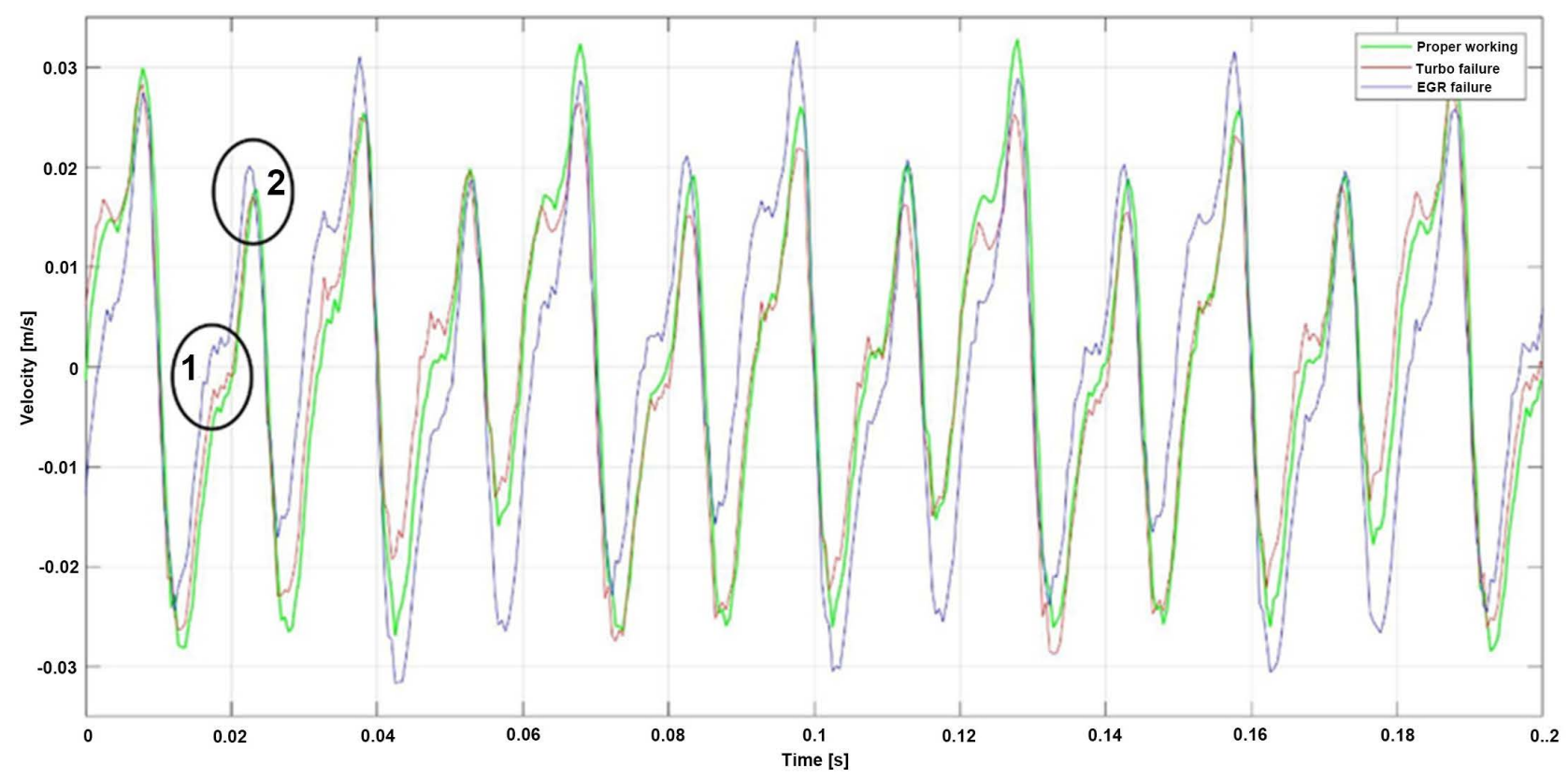

Figure 1. Engine vibrations, measured on the head in three conditions (solid line—engine in good working order, dashed-damaged turbocharger, dotted-damaged EGR system), $2000 \mathrm{rpm}, 50 \mathrm{Nm}$.

during pre-injection, the instability of the system (oscillations) can be observed, which is a typical phenomenon. Moreover, the engine with a damaged EGR system vibrates with a greater amplitude (as compared to a well-functioning engine), and with a damaged turbocharger-with a smaller one.

The source signals were subjected to the wavelet decomposition process. Below (Figure 2) the signal recording and its approximation at level 3 are presented.

Individual decomposition coefficients are presented in Figure 3.

Selecting the right level of decomposition becomes vital, especially if the decomposition is to return valuable (from a diagnostic point of view) coefficients. Haar's wavelet on level 3 allows for implementing this type of diagnostics for most diagnostic systems based on modern signal and line processors.

To make the diagnostic message readable, a package wavelet analysis was introduced. The initiating tree is shown in Figure 4.

The initiating tree is extremely helpful while analysing results. It allows you to preview every step of the analysis (so you can call it the "wavelet analysis debugger"). Of course, its online use is limited.

Package analysis is popular mainly because of its graphical presentation of results. Figure 5 shows the result of the wavelet package decomposition of an engine in good working order.

Comparison of the most characteristic (graphical) parts of the transform result allows us to find differences in the values of decomposition coefficients. However, it is difficult, and requires careful measurement and proper selection of the level of decomposition (Figure 6). 


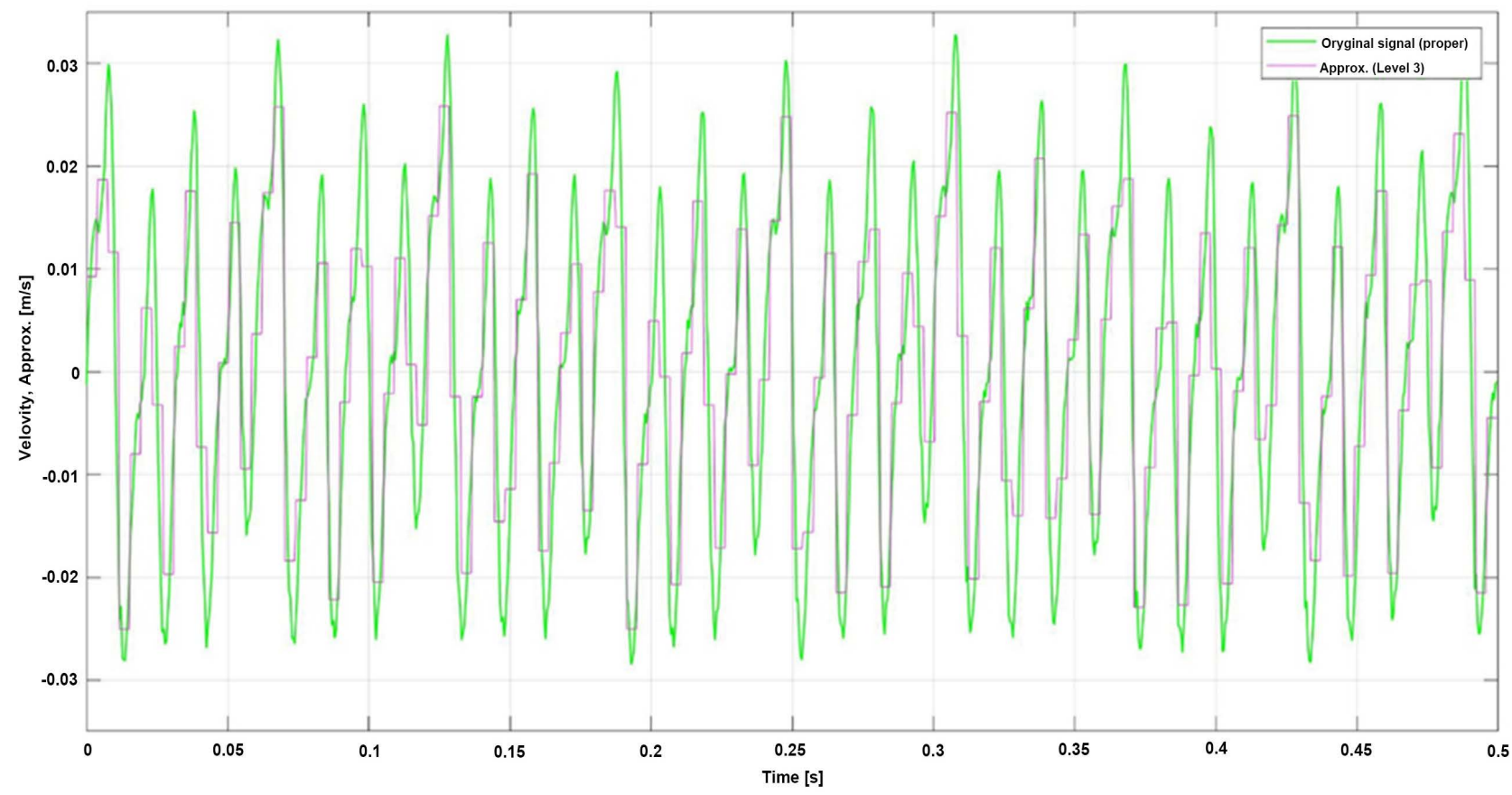

Figure 2. Vibration speed of a properly functioning engine and its approximation (level 3, Haar).

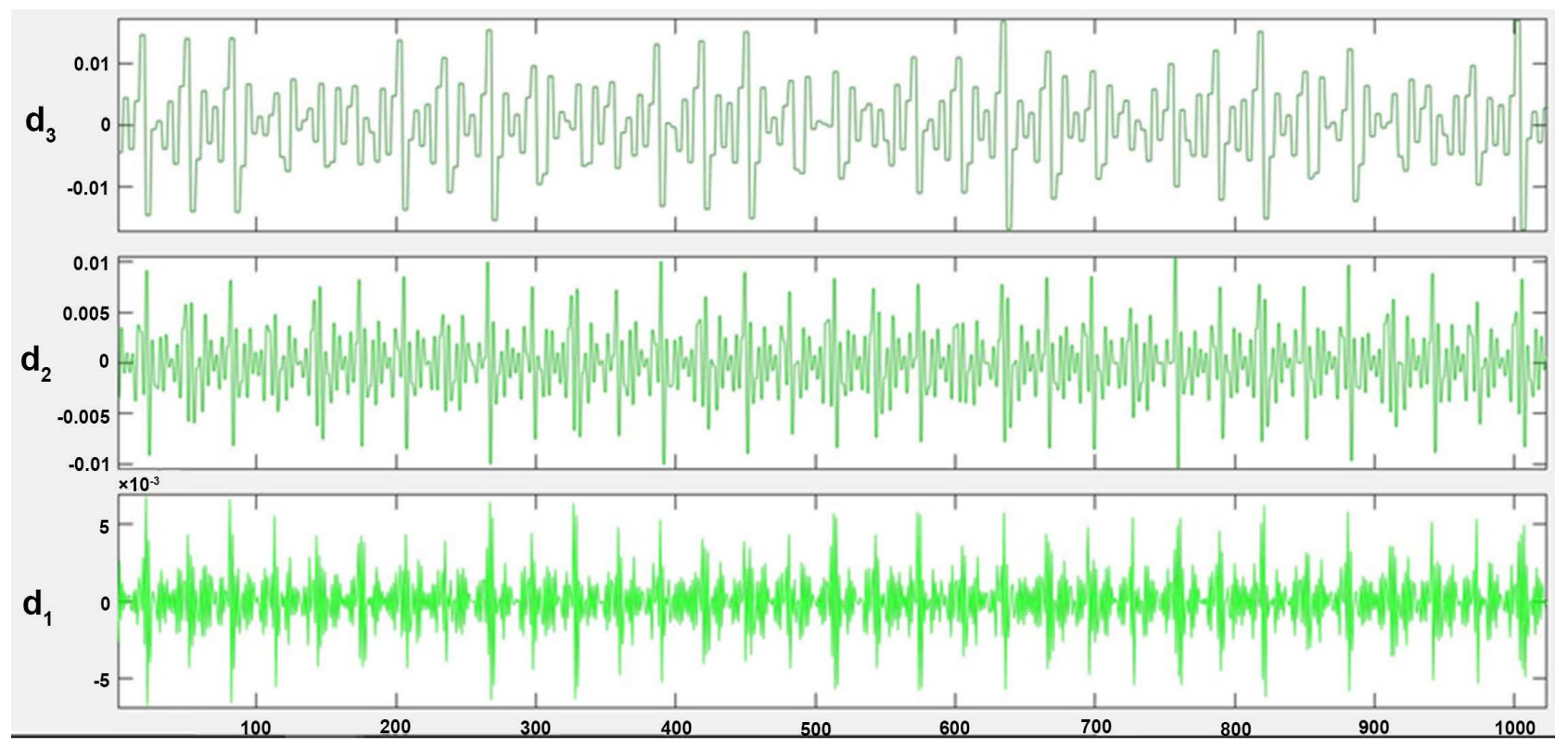

Figure 3. Decomposition coefficients of the vibration speed signal of a properly operating engine (level 3, Haar).

The presented drawings show the way of decomposing the motor in various states. Figure 5 shows the entire decomposition obtained from the wavelet analysis of a fully functional engine. Figure 6 contains a comparison of the obtained diagnostic information of the engine for 3 states (1-efficient engine, 2-with a damaged turbocharger, 3-with a damaged EGR system). In this figure, only the last decomposition levels are shown so that you can perform a comparison analysis that indicates large differences in individual states. 


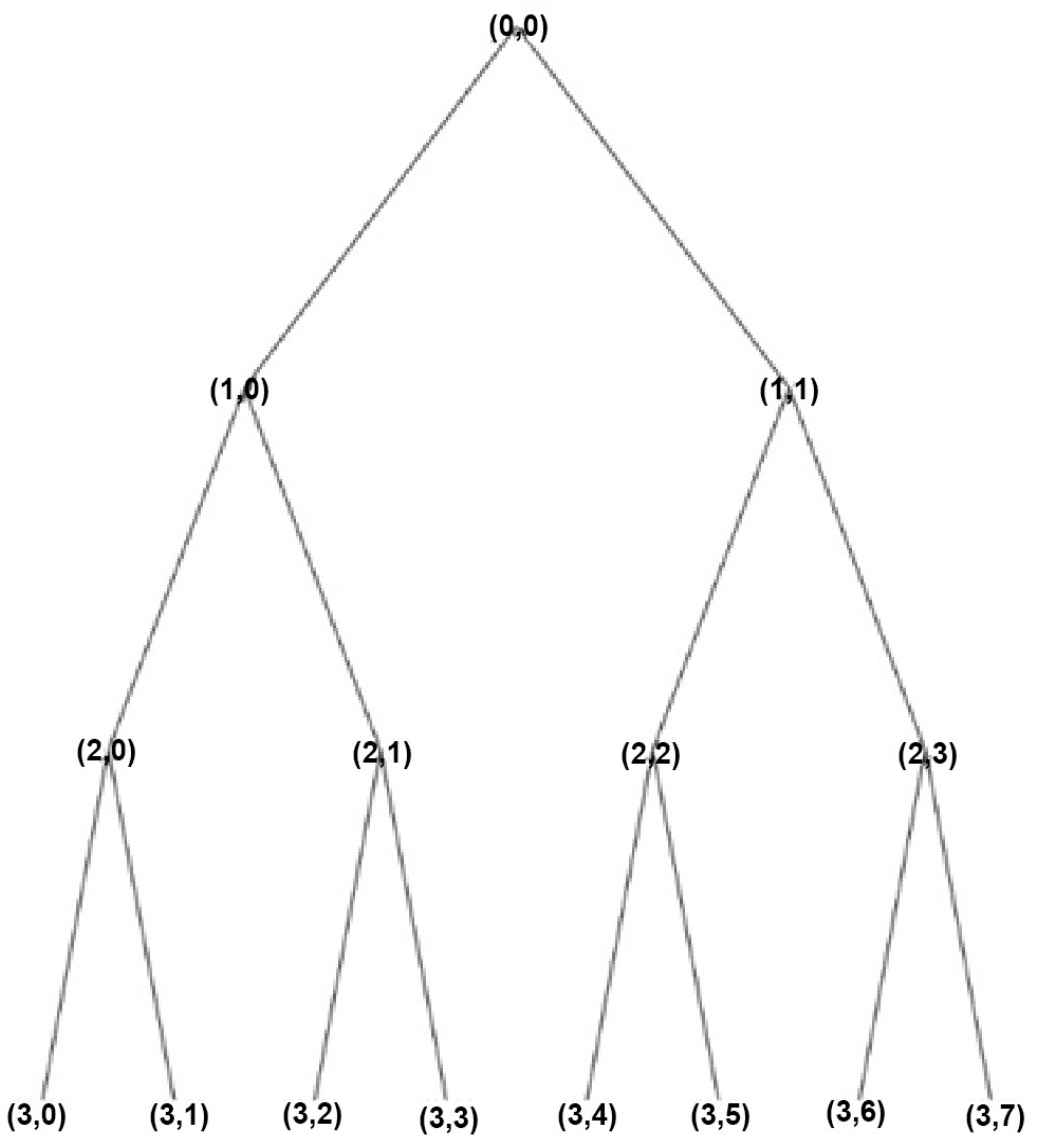

Figure 4. Initiating tree for package wavelet analysis (level 3, Haar).

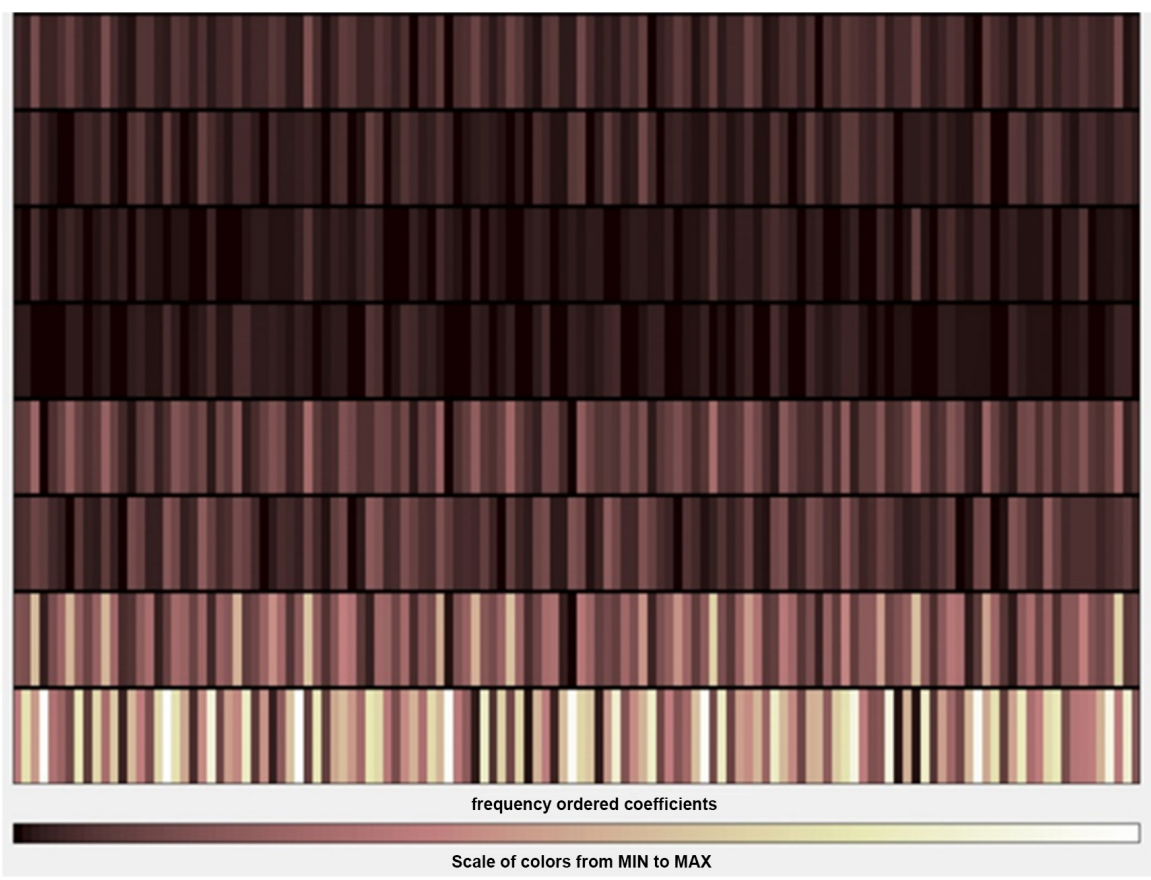

Figure 5. Package wavelet analysis of well-functioning engine vibrations (2000 rpm, 50 $\mathrm{Nm})$. 

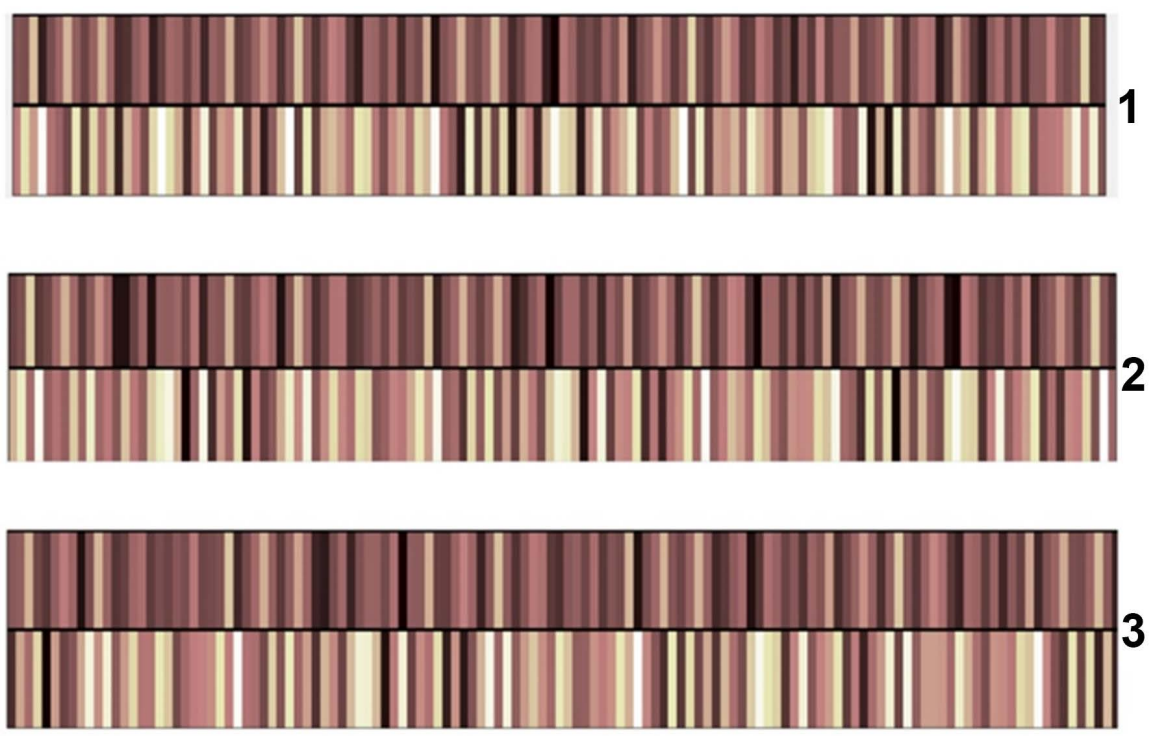

Scale of colors from MIN to MAX

Figure 6. Package wavelet analysis of well-functioning engine vibrations (1), with a damaged turbocharger (2) and damaged EGR system (3).

\section{Results and Discussion}

Despite visible differences (Figure 6), the question remains how useful the wavelet transform in the analysis can be. The above data show differences for individual engine conditions. However, in order to directly determine how big the difference is, the authors propose to use the graphical "divide" function (Figure 7) between particular results. This feature is available in many graphics programs (in this case: Photoshop CS6). Its use in this case gives very positive results directly visible in the form of darkened fields (or not). In other words, the function, after applying the layers, allows indicating places in which individual coefficients differ (black colour).

Even more unambiguous results can be obtained if the original signal is windowed before being decomposed. The window with the best reproduction of amplitude, high dynamics and low resolution, was used. It was previously shown that it is the flattop window (for relative speeds) [19]. The results are shown below.

Wavelet analysis is commonly used in cardiac imaging, for instance. Doctors look for changes in the functioning of the heart (whose operation is, after all, "stationary" in its nature). Division into a stationary or non-stationary signal loses its significance from the statistical point of view, along with the increase in computational power of diagnostic systems.

The authors stated that diagnostics using wavelet analysis can be the basis for a thorough analysis of the technical condition of machines. With less precise measurements (acceleration measurements in this case), lower level of decomposition is required; however, the result will be limited to only a few "major" 


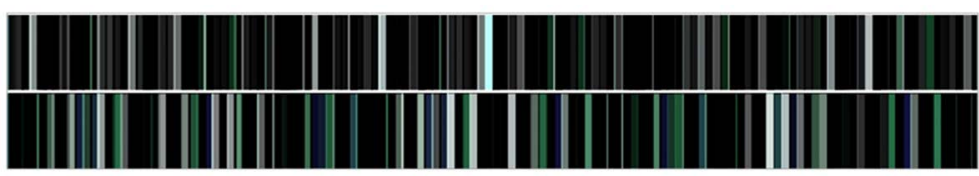

Properly working (divide) EGR failure

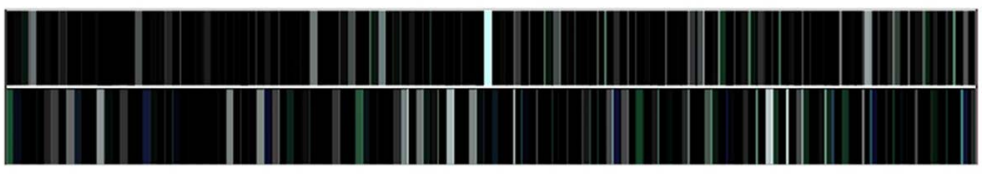

Properly working (divide) Turbo failure

Figure 7. The result of the application of the graphical divide function between the layers, being the results of the wavelet analysis; black indicates the places that differ.

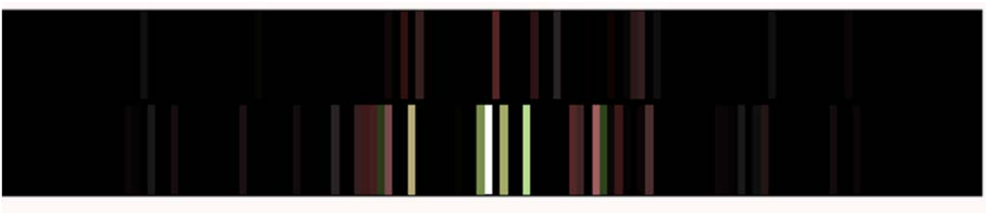

Properly working (divide) EGR failure (windowing)

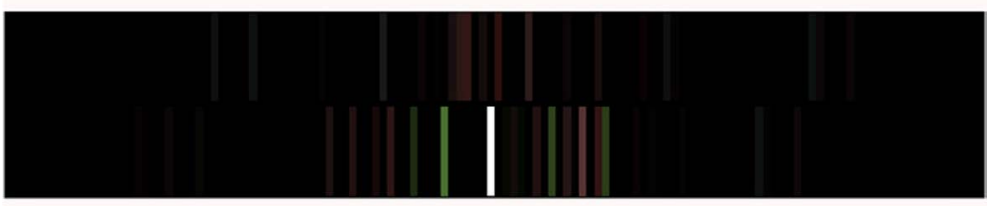

Properly working (divide) Turbo failure (windowing)

Figure 8. The result of the graphical divide function application between the layers, being the results of the wavelet analysis; black indicates the places that differ; original signals were subjected to windowing (Flattop).

changes in the condition of the tested unit. In the case of testing based on vibrometric laser systems, it is possible to diagnose less obvious defects. An example here may be the damage to the EGR system, which, as has been shown (Figure 7), is marked by significant changes in the value of coefficients, although not as large as in the case of damage to the recharge system.

Additional signal processing operations (such as windowing, and in this case, a flattop window) allows us to clearly distinguish the changes presented by means of package decomposition. In this case (Figure 8), the differences in particular parts of the imaging are clearly visible, which is a direct derivative of the condition of an engine.

A multidimensional function ought to become the basis for vibroacoustic diagnostics of engines. Apart from its size, time and frequency, the function will introduce an additional operating feature, i.e. rotational speed or torque.

\section{Declarations}

The materials presented in the publication can be used in other studies and 
presented in the articles. The authors declare that they have no competing interests. The presented research was not carried out under any grant. The research was carried out as part of the statutory work of the research unit (Wroclaw University of Science and Technology, Mechanical Dept., Division of Automotive Eng.). The contribution of Radoslaw Wrobel (correspondence author) was based on the performance of research and their processing and analysis. The contribution of Andrzej Kazmierczak is based on the performance of research, analysis and editing of the text. The authors confirm that all works related to the presented article are original and have been made only by them.

\section{Acknowledgements}

Research financed from Wroclaw University of Science and Technology statutory funds no. 0401/003/18.

\section{Conflicts of Interest}

The authors declare no conflicts of interest regarding the publication of this paper.

\section{References}

[1] Daubechies, I. (1992) Ten Lectures on Wavelets. SIAM, Philadelphia. https://doi.org/10.1137/1.9781611970104

[2] Ingle, V.K. and Proakis, J.G. (2015) Digital Signal Processing Using MATLAB: A Problem Solving Companion. 4th Edition, Cengage Learning, Boston.

[3] Mertins, A. (1999) Signal Analisys: Wavelets, Filter Banks, Time Frequency Transforms and Applications. Wiley, New York.

[4] Lyons, R.G. (2004) Understanding Digital Signal Processing. Pearson Education, London.

[5] Eadie, W.T., Drijard, D., James, F.E., Roos, M. and Sadoulet, B. (1971) Statistical Methods in Experimental Physics. North-Holland, Amsterdam.

[6] Hammond, J.K. and White, P.R. (1996) The Analysis of Non-Stationery Signals Using Time-Frequency Methods. Journal of Sound and Vibration, 190, 419-447. https://doi.org/10.1006/jsvi.1996.0072

[7] Matko, V. and Jezernik, K. (2010) Greatly Improved Small Inductance Measurement Using Quartz Crystal Parasitic Capacitance Compensation. Sensors, 4, 3954-3960. https://doi.org/10.3390/s100403954

[8] Matko, V. (2011) Next Generation AT-Cut Quartz Crystal Sensing Devices. Sensors, 5, 4474-4482. https://doi.org/10.3390/s110504474

[9] Delvecchio, S., Bonfiglio, P. and Pompoli, F. (2018) Vibro-Acoustic Conditio Monitoring of Internal Combustion Engines: A Critical Review of Existing Techniques. Mechanical Systems and Signal Processing, 99, 661-683. https://doi.org/10.1016/j.ymssp.2017.06.033

[10] Salivahanan, S. and Gnanaptiya, C. (2011) Digital Signal Processing. McGraw-Hill Education, New York.

[11] Rao, S.S. (1995) Mechanical Vibrations. Addison-Wesley, Boston.

[12] De Silva, C. (2000) Vibrations Fundamentals and Practice. CRC Press, New York. 
[13] Kelly, S.G. (2000) Fundamentals of Mechanical Vibrations. McGraw-Hill Education, New York.

[14] William, J.P. (2011) Mechanical Vibration. Wiley, Hoboken.

[15] Howard, G. (2015) The Physics of Waves. Prentice Hall, Upper Saddle River.

[16] Singiresu, R.S. (2005) Mechanical Vibrations. Prentice Hall, Singapore.

[17] Bosch Technical Library (2004) Control Systems of Diesel Engines. WKŁ, Warszawa.

[18] Dudzik, G., Wąż, A., Kaczmarek, P., Sotor, J., Krzempek, K., Soboń, G.J., Antończak, A. and Abramski, K. (2011) Multichannel Flexible Fiber Vibrometer. Defense, Security, and Sensing 2011: Emerging Technologies and Laser Sensors and Systems, Orlando, 2011, 289-290.

[19] Singiresu, S. (2005) Rao: Mechanical Vibrations. Prentice Hall, Singapore.

[20] Kaźmierczak, A., Reksa, M., Moczko, P. and Wróbel, R. (2015) Comparative Analysis of the Vibrations of a Spark-Ignition Engine with and without Supercharging, Mounted in New Motor Vehicles. Journal of Kones, 18, 443-448.

[21] Mallat, S. (1989) A Theory for Multiresolution Signal Decomposition: The Wavelet Representation. IEEE Transactions and Pattern Analysis and Machine Intelligence, 11, 674-693. https://doi.org/10.1109/34.192463

[22] Mertins, A. (1989) Signal Analysis: Wavelets, Filter Banks, Time Frequency Transform and Applications. Wiley, Hoboken

\section{Abbreviations}

$\begin{array}{cl}\text { LDV } & \text { Laser Doppler Vibrometry } \\ \text { TDC } & \text { Top Dead Centre } \\ \text { EGR } & \text { Exhaust Gas Recirculation } \\ \text { DWT } & \text { Discrete Wavelet Transform }\end{array}$

